\title{
De los apuntes al esquema. Repensando a Francisco Miró Quesada y en defensa de una razón limitada y pragmática
}

\author{
From the notes to the scheme. Rethinking Francisco Miró Quesada \\ and in defense of a limited and pragmatic reason
}

\author{
Richard Orozco C. \\ Universidad Nacional Mayor de San Marcos, Lima, Perú \\ Contacto: richard.orozco@unmsm.edu.pe \\ https://orcid.org/0000-0001-9655-1322
}

\section{Resumen}

En este artículo, el autor muestra una tensión al interior de la obra de Francisco Miró Quesada. Una tensión que aparece en su lucha contra los escépticos y en su intento por salvar una razón mínima. Dicha tensión se manifiesta tras la publicación de los Apuntes en 1963 y luego en el Esquema en 2012. Obviamente, la tensión recorre esos 50 años. Pero el autor también añade lo que pudo haber sido una salida a dicha tensión: el reconocimiento y valoración de una razón limitada y pragmática. Así, frente a la propuesta de Miró Quesada de una razón mínima con la cual salvar el proyecto racionalista, el autor propone una visión pragmatista de la razón, que sería el reconocimiento de las consideraciones evolucionistas.

Palabras claves: Francisco Miró Quesada; Racionalismo; Razón mínima; Razón limitada; Razón pragmática.

\begin{abstract}
In this article, the author shows a tension within the work of Francisco Miró Quesada. A tension that appears in his fight against the skeptics and in his attempt to save a minimal reason. This tension manifests itself from the publication of the Apuntes in 1963 and then in the Outline in 2012. Obviously, the tension goes through those 50 years. However, the author also adds what could have been an outlet to this tension: the recognition and valuation of a bounded and pragmatic reason. Thus, faced with the proposal of Miró Quesada for a minimal reason with which to save the rationalist project, the author proposes a pragmatic view of reason that would be the recognition of evolutionary considerations.
\end{abstract}

Keywords: Francisco Miró Quesada; Rationalism; Minimum Reason; Bounded Reason; Pragmatic Reason.

Recibido: 23.04 .19

Aceptado: 18.09.19 


\section{Introducción}

En 1963, Francisco Miró Quesada (en adelante FMQ) publicó un texto titulado Apuntes para una teoría de la razón (2012d). Dicho texto había sido precedido por un artículo con el mismo título publicado un año antes en la revista Dianoia (Miró Quesada, 2012c). En el año 2012 apareció un nuevo texto suyo, menos pretencioso y mucho más tímido que el anterior y que esta vez se titulaba Esquema para una teoría de la razón (2012g). Mi propuesta busca interpretar este paso desde los Apuntes hasta el Esquema. Las preguntas que me guían son: ¿qué buscaba el autor detrás de dicha travesía? ¿Hasta qué punto se consiguió lo que se buscaba? ¿Qué cambios se han producido durante dicha travesía?, y ¿qué permaneció incólume a pesar de los años? Mi interés es buscar responder a estas preguntas para luego desarrollar una idea que en mi título se enuncia con transparencia: la defensa de una razón limitada y pragmática.

La tesis principal que desarrollaré es que FMQ siempre tuvo frente a sí el principal rasgo de la razón, aunque por una cuestión vital se resistió constantemente a asumirlo. Ese principal rasgo que se revelaba era el carácter limitado de la razón que, sin embargo, FMQ nunca quiso asumir debido a una cuestión vital y pragmática. Desde el mismo momento en que los tituló como 'Apuntes' y más tarde como 'Esquema', siempre supuso lo limitado de sus logros. Nunca consideró que él pudiera culminar una teoría completa de la razón y de hecho tomó conciencia, en su época de madurez, de que quizá dicha empresa era para ser realizada por un equipo y no para una mente individual. Lo limitado de sus resultados era una consciencia transparente en su reflexión; sin embargo, lo que nunca se atrevió a hacer fue definir la razón misma como limitada. Es decir, él era consciente de que su trabajo era limitado, que el proyecto excedía sus fuerzas, pero no quiso definir a la razón como limitada. Evidentemente esto parece deberse a que tal decisión lo hubiese convertido, ante sus propios ojos, en uno más de los escépticos contra quienes tanto luchó. Así pues, se refleja una tensión en la obra de Miró Quesada, una lucha entre la consciencia cada vez más clara de lo limitada que es la razón, pero una necesidad por querer salvar al racionalismo en su lucha contra el escepticismo. En este ensayo busco aclarar 
dicha tensión, sus causas y consecuencias. Todo esto, sin embargo, me permitirá ir desarrollando una defensa de lo que yo entiendo por razón limitada. Finalmente, en respuesta al análisis que hace FMQ respecto de lo que él llama una nueva forma de escepticismo, refiriéndose al pragmatismo, intentaré hacer una defensa de este, mostrando el parecido de familia que hay entre esta razón limitada y el pragmatismo. Mi objetivo es pues interpretar la tensión en la que siempre estuvo inserto el pensamiento de FMQ, que era menos clara en los años sesenta, pero que se aclaró más para la primera década del 2000.

\section{El contexto de la tensión}

En el artículo titulado "Esbozo de mi posición filosófica" que data de 1953 (Miró Quesada, 2012a), ya FMQ mostraba con claridad cuál era el contexto que enfrentaba y la interrogante que debía responder. Dicha presentación no dista mucho del artículo publicado en Dianoia de 1962 y del libro Apuntes de 1963. Lo que deja en claro no es solo que dicho contexto le es evidente, sino que además lo interpela de una manera significativa. Voy a explicitar dicho contexto con cuatro características que FMQ reconoce y enuncia.

La primera circunstancia que hay que considerar es la aparición de teorías científicas realmente revolucionarias. Julián Marías definió a dicha época, finales del siglo XIX y comienzos del XX, como el "derrumbe de la física clásica" (1964); FMQ considera que la situación alcanza a toda la ciencia y no solo a la física, y por ello prefiere hablar de una "crisis de la ciencia" (2012b). Siendo más específicos, FMQ habla de tres grandes crisis de la ciencia. En primer lugar está el advenimiento de las geometrías no euclidianas que, según nuestro autor, interpela de una manera significativa la confianza que matemáticos y filósofos habían puesto en la evidencia de los postulados de Euclides. Luego tenemos el descubrimiento de la paradoja de Burali-Forti que permitió dudas incisivas sobre los métodos matemáticos tradicionales. En tercer lugar irrumpe la teoría de la relatividad que tiene como principal consecuencia epistemológica la exigencia para someter a una severa revisión tanto el principio de causalidad como el de substancia. Sin embargo, el caso más notable según FMQ, o mejor dicho la revolución más 
significativa, sucede a partir de los trabajos de Kurt Gödel. Aunque podría parecer que esta revolución solo sucede en el ámbito de la lógica o de la matemática, y FMQ es prudente antes de hacer cualquier extrapolación anticipada, es cierto sin embargo que los teoremas de Gödel han realmente provocado toda una revolución en las posibilidades de la ciencia. La indecidibilidad, la inconsistencia o la inderivabilidad pueden ser rasgos innegables de las teorías de las matemáticas clásicas y, a partir de ello, de las ciencias que de ellas dependen.

Frente a esta primera circunstancia, Miró Quesada reconoce una segunda característica del contexto que enfrenta. Se trata de la incapacidad de los modelos epistemológicos clásicos para responder a dicha crisis de la ciencia. Por modelos clásicos de la epistemología, FMQ entiende racionalismo, empirismo, historicismo, pragmatismo y escepticismo. En sus palabras, se trata de un "rebasamiento de los marcos tradicionales". El racionalismo, dice, es el que más herido ha quedado, pues es evidente que sus principales postulados han sido falseados. Su ideal, ambicioso y radical, ha fracasado al demostrarse la imposibilidad de la autofundamentación absoluta de las ciencias. El caso del empirismo es también un fracaso, pues el desarrollo de las ciencias formales ha demostrado "la imposibilidad de reducir el conocimiento matemático a una pura abstracción de cualidades y relaciones de entes concretos" (Miró Quesada, 2012d, p. 197). La suerte del pragmatismo no es distinta, su fracaso es evidente cuando es incapaz de demostrar que todo conocimiento deriva de una eficacia práctica; y nuevamente es paradigmático el caso del conocimiento lógico matemático, pues no hay forma de derivar los postulados lógicos de tal esquema mundano. Como dice FMQ, "Una teoría matemática y lógica presenta una fuerza interna que no depende de ninguna eficacia” (2012d, p. 201). En el caso del escepticismo, se trata más bien de un término general que, según nuestro autor, engloba tanto al pragmatismo como al historicismo y a muchos movimientos filosóficos contemporáneos. Lo común de ellos es que todos dudan de las posibilidades de la razón o de su capacidad para lograr sus más altos objetivos. En los Esquemas, publicados el año 2012, FMQ define a todos esos modelos 
escépticos como manifestaciones de una razón impura, y le dedica a esta una gran sección del libro. Una definición general de estos escépticos dice que son los que sostienen que es imposible saber si se puede o no fundamentar racionalmente nuestros conocimientos (Miró Quesada, 2012g, p. 38). Con dos notas quisiera comentar las expresiones de FMQ respecto de los escépticos. En primer lugar, cuando nuestro autor observa el alcance del escepticismo, lo reconoce desde los pirrónicos hasta el segundo Wittgenstein. De Wittgenstein dice que las sutilezas y profundidad de sus argumentos muestran una nueva vida para el escepticismo, de allí la importancia de discutir las tesis presentadas en Investigaciones filosóficas (Wittgenstein, 1988). También es resaltable el hecho de que FMQ ve un canto de victoria demasiado apresurado por parte de todos los escépticos contemporáneos. Este es un aspecto importante que discutiremos más adelante, pero que vale la pena reconocer como parte de este contexto. Lo que se está resaltando es la manera en cómo la mayoría de los modelos escépticos contemporáneos - historicismos, pragmatismo o relativismos - ven la situación de crisis del racionalismo como una expresión de su victoria definitiva. Miro Quesada nota ese canto de victoria anticipado y más bien lo reconoce como parte de este contexto más general.

Como tercer rasgo de este contexto que FMQ enfrenta, aparece la nueva situación de la filosofía en donde, según nuestro autor, "gracias al método simbólico matemático", la lógica y la epistemología han alcanzado "un nivel de rigor nunca antes igualado en la historia de la filosofía" (Miró Quesada, 2012a, p. 89). Así pues, FMQ está haciendo eco del sentir que Hans Reichenbach manifiesta en su libro The Rise of Scientific Philosophy (1967) que había sido publicado en 1951 y en el cual se resaltaba la nueva situación de la filosofía en consonancia con la misma idea que presenta FMQ. Hay que anotar, además, que este sentir de Reichenbach no es exclusivo de él, pues quizá es común entre los miembros del Círculo de Viena y compartido por los llamados positivistas lógicos. De allí se entiende la admiración que FMQ profesa hacia autores como Russell, Whitehead, Hilbert y el propio Wittgenstein, quienes realizaron aportes importantes hacia un tipo especial de lenguaje filosófico: riguroso, formalizado y en diálogo con las ciencias. No obstante, es necesario precisar que, aunque FMQ 
admiraba ese estilo de hacer filosofía y aunque él mismo produjera textos de ese nivel de rigor en las formalizaciones, no por ello desmerecía lo que él llamaba la 'filosofía literaria' (Miró Quesada, 1966). FMQ vio entre estos últimos a insignes filósofos peruanos por quienes guardó siempre una enorme admiración. Entre ellos destacaba a Alejandro Deustua y a Mariano Iberico, siendo este último uno de sus profesores por quien más cariño sentía. Era pues perfectamente consciente de esta bifurcación que se abría en la filosofía, pero vio más bien posibilidades en ella y no razones para el menosprecio o la exclusión.

Finalmente, y como parte de este contexto que estamos comentando, FMQ consideró el supuesto derrumbe de la razón. Este supuesto derrumbe se presenta con varios rostros: fin de la ciencia, fin de la metafísica, fin de los metarrelatos o la posfilosofía, etc. Era claro para FMQ que el racionalismo, con su pretenciosa agenda, ya no podía sostenerse; pero no era tan evidente para nuestro filósofo que esto nos condujera a la imposibilidad de un discurso trascendental o hacia la muerte de los absolutos. Si el racionalismo no sobrevive, por lo menos en su versión clásica, eso no significa que sea imposible el conocimiento absoluto o que la razón se haya derrumbado. Sin embargo, eso es lo que se publicita en un tipo de discurso que se difunde en nuestra cultura contemporánea en la que los escépticos cantan victoria. Una cara de este rasgo que comentamos es justamente eso, es decir, que distintas caras del escepticismo se presentan como victoriosas. El relativismo o el historicismo reclaman anticipadamente un triunfo sin haber reconocido ampliamente lo que sucede en la cultura. Entonces, para FMQ ese sonado derrumbe de la razón no es sino un alarde exagerado producto de un análisis poco riguroso respecto de lo que sucede en la cultura contemporánea.

Así pues tenemos el panorama completo con estos cuatro rasgos de un contexto que le fue claro a nuestro autor desde los años sesenta y que no cambió grandemente hasta la primera década del 2000. La crisis de la ciencia, la incapacidad de los modelos epistemológicos clásicos para comprender dicha crisis, la rigurosidad que ha alcanzado la filosofía y finalmente una exagerada publicidad de un supuesto derrumbe de la razón, todas esas cuatro características 
explican la realidad cultural que FMQ debía enfrentar y desde la cual buscaba obtener una respuesta satisfactoria.

\section{La tensión en las reflexiones sobre la razón}

Hablo de 'tensión' en el pensamiento de FMQ, pues desde los Apuntes, o incluso antes de eso, en el texto "La crisis de la ciencia" de 1953, ya parecía claro el problema que enfrentaba y no vio cambiar ese panorama hasta la publicación del Esquema, que recién sucedió en el año 2012. Era un panorama que interpelaba al filósofo buscando de él una respuesta contundente sobre la razón; pero esa respuesta definitiva nunca llegó. Hay más bien un panorama de respuestas que muestran una tensión en la que no es posible una respuesta definitiva a los problemas planteados, pero al mismo tiempo se impone la imposibilidad de aceptar que esa filosofía impura, la de los escépticos, sea la que haya triunfado. Consideremos mejor la imagen de la tensión que estamos advirtiendo.

Esta tensión a la que nos referimos presenta un anverso y un reverso: por un lado, la necesidad de responder contundentemente al contexto de crisis; por otro, la imposibilidad de declarar la victoria del escepticismo. Voy a aclarar cada uno de los lados de la tensión, con apoyo de textos, mostrando el panorama de la respuesta de FMQ para hacer claro que se trata de una tensión y no de una respuesta definitiva. Sin embargo, antes deseo presentar una interpretación de cómo veo yo la presencia de una tensión al interior de un sistema de pensamiento.

Algunos podrían pensar que la tensión en un sistema de pensamiento puede ser signo de debilidad, timidez o incapacidad, mas ese no es mi caso. Yo considero, en cambio, que la tensión es signo de una honradez intelectual mayúscula. Creo que los más interesantes sistemas de pensamiento en la historia han sido erigidos sobre la base de una tensión de opuestos que eran por sí solos inaceptables. El filósofo que revela la tensión es aquel que descubre su propio límite y que de alguna forma va descubriendo el límite propio de la razón. La razón se nos aparece así como un movimiento dialéctico y no como un esquema fijo de verdades absolutas. El filósofo puede sentir la necesidad de escapar de la tensión, pues esta le genera incertidumbre, pero dicha fuga solo le es posible 
sobre la base de una apuesta pragmática o sobre el encubrimiento de la tensión. Por el contrario, la honestidad intelectual obliga a convivir con la incertidumbre y aceptar la perenne tensión.

¿Por qué no declarar entonces la victoria del escepticismo? Si lo que vemos ante el contexto que hemos descrito es por doquier muestras de lo limitada que es la razón, ¿por qué no aceptar entonces que verdaderamente los escépticos han acertado? ¿Por qué FMQ comenzó su texto de 2012, el Esquema, dedicando toda la primera parte a desarrollar una defensa amplia frente al escepticismo? Parece una defensa inútil, pues lo que se observa es más bien el reconocimiento de las limitaciones de la razón. Es claro, pues, que el enemigo de quien había que cuidarse, desde los primeros escritos en la década de 1950 hasta la publicación del Esquema, es el escepticismo. ¿Por qué? Creo yo haber entreleído la respuesta: el escepticismo no puede vencer, porque sino quien muere es la misma filosofía. La expresión clara aparece en un texto de 1995 que se tituló "Autobiografía filosófica". En ese texto leemos la siguiente sentencia: "Si los escépticos tienen la razón, la filosofía se transforma en una jerigonza ininteligible que hay que botar al tacho de basura” (Miró Quesada, 2012f, p. 127).

Es decir, si quienes declaran el derrumbe de la razón estuvieran en lo cierto; si la razón es incapaz de lograr un conocimiento seguro, certero y absoluto y que a la vez sea autofundado, no meramente intuitivo; si eso es cierto, entonces la que se derrumba es la filosofía misma y sus más nobles objetivos. Quiero resaltar entonces que la lucha no se desarrolla en el ámbito teórico, enfrentando argumentos deductivos, la lucha es de carácter práctico-vital. El problema tiene que ver con el corazón de la vida misma del filósofo. La tensión que enfrenta FMQ es una tensión vital que amenaza su proyecto de vida.

Podría parecer que el argumento con que FMQ enfrenta al escepticismo fuese un argumento deductivo, pero esto no es así. Como trataré de demostrar, el argumento es claramente de tipo pragmático. Cuando uno lee el Esquema y ve la defensa que realiza FMQ frente a los escépticos, en cualquiera de sus variedades, uno encuentra que el principal argumento es que los escépticos no pueden vencer 
ya que ellos defienden una especie de filosofía impura. FMQ explica esa impureza como el intento de una razón que deslegitima a la razón misma. Los escépticos, los que pregonan el derrumbe de la razón, en el fondo lo que están intentando hacer es mostrar, con la razón, la incapacidad de la propia razón. En sus propias palabras,

[u]tilizar la razón para demostrar la imposibilidad del conocimiento racional, es usarla contra su naturaleza. Quien la usa está haciendo trampa a sí mismo y a los demás. Es como si quien utilizara su razón no fuera puro en sus intenciones cognoscitivas. Pero, por más que haga, no puede matar a la razón, pues tiene que utilizarla para cometer el asesinato: la razón no puede hacerse el hara-kiri. (Miró Quesada, 2012g, p. 42)

FMQ considera a este un argumento circular y lo ve como su principal defensa frente a los escépticos. Todos quienes se han enfrentado a los escépticos han usado o este o un segundo argumento: la evidencia absoluta de muchas verdades que son incuestionables, principalmente en las ciencias formales (Miró Quesada, 2012g, p. 42). Además, es interesante también comentar aquí el realce que FMQ hace del argumento elaborado por Ortega y Gasset contra los escépticos. Este había concluido que si los escépticos afirman la imposibilidad de llegar a la verdad, primero deben conocer la verdad para poder reconocer que no la pueden alcanzar. "El relativismo es, a la postre, escepticismo, y el escepticismo, justificado como objeción a toda teoría, es una teoría suicida” (Ortega y Gasset, 1966).

Nos quedan dos cuestiones a tratar: a) cómo es que este argumento contra los escépticos puede entenderse como un fundamento pragmático; y b) por qué la victoria de los escépticos significaría la muerte de la filosofía. En el primer caso, debemos reconocer que el argumento de FMQ dice algo así: la razón no puede derrumbar a la razón. El filósofo está mostrando una falacia en el argumento escéptico, una falacia de circularidad. Si el escéptico debe usar la razón, y no hay otra alternativa, entonces no puede demostrar la incapacidad de la misma razón, pues en ese caso caería en una paradoja.

No obstante, observemos bien y veamos formas en que no resulte paradójico el argumento. Propongo tres consideraciones en las que el argumento 
no resulta paradójico y más bien puede derivar en idóneo. En primer lugar, si consideramos que en las dos menciones de la razón que presenta el argumento se están presentando dos actividades distintas, si el primero hace referencia a la razón en tanto agente y el segundo se refiere a la razón en tanto pasiente (pasivo), ya se elimina la circularidad. Lo que tendríamos es una razón que con sus armas y desde adentro define las posibilidades de la razón objetivizada. En segundo lugar, podríamos considerar que la primera razón hace referencia a la razón con pretensiones descriptivas, mientras que la segunda hace referencia a una razón con pretensiones normativas. Entonces el argumento se convierte en algo así: la razón descriptiva no puede demostrar la incapacidad de la razón normativa. Pero ¿por qué no podría ser así? ¿No podría acaso la razón hacer un reconocimiento de sus armas y posibilidades para ver el verdadero alcance de sus pretensiones normativas? Sí podría. Es lo que han intentado hacer muchos filósofos desde Kant, reconociendo los límites de la propia razón en sus ideales más altos. Por último, pudiéramos reconocer planos distintos en los que se usa la palabra razón en el argumento, diferenciando la razón como metalenguaje y la razón como lenguaje objeto. Nuevamente se desmorona la paradoja.

Lo desconcertante es que FMQ, siendo un lógico de primer nivel, bien podría haber reconocido estas formas de salvar la supuesta paradoja, pero no lo hizo. A menos que el argumento no sea lógico sino pragmático, como estoy tratando de mostrar. Entonces, el centro del argumento 'la razón no puede demostrar la incapacidad de la razón' no está en la paradoja que se forma por el doble uso del concepto 'razón', sino más bien, en el 'no puede'. La razón no puede derrumbar la razón, los escépticos no pueden vencer, pues la que pierde es la filosofía. Es, como dije, una tensión vital que subyace al interior del proyecto del filósofo.

Según nuestro autor, el sentido profundo de lo que es filosofía se juega en la lucha entre el racionalismo y el escepticismo. Si el escepticismo vence no hay sitio para la filosofía, habría que admitir más bien, como alguna vez lo anunció Richard Rorty (1996), el advenimiento de un mundo posfilosófico. Ese sería un mundo en el que no hay posibilidad de hablar sobre principios fundamentales 
y eso es justamente la muerte de la filosofía, pues para FMQ la filosofía es teoría de los principios. Si el escepticismo ha vencido, cree nuestro autor, no podría reconocerse principios fundamentales en cada uno de los ámbitos donde necesitamos de la razón una respuesta definitiva: en las ciencias sociales, en la política, en la comprensión de la historia, etc. Si el escepticismo vence, entonces solo nos queda una casuística y el caso particular.

Entendamos, pues, que el problema es vital y pragmático. En la posibilidad misma de que no haya forma definitiva de poder acercarse a los principios fundamentales de la razón o de que dichos principios no existan, en la posibilidad misma de que tales principios sean solo una ilusión más y que los escépticos tengan razón; entonces habremos confirmado el fin de ese tipo de cultura especial que Husserl (2008) denominó cultura filosófica. Ese peligro que Husserl veía llegar con el positivismo, FMQ lo ve acercarse con los escépticos.

Cuando FMQ escribe su Esquema para una teoría de la razón — no una teoría de la razón, pues no cree concebirla plenamente-, él considera en dicho esquema la presentación de los principios de la razón. Así define como principios de la razón: el principio de no-arbitrariedad, el principio de seguimiento, el principio de creatividad y el principio de unidad. El desarrollo de estos principios, su defensa y reconocimiento, son su verdadera lucha contra el escepticismo. No obstante, como él mismo lo planteó, es solo el principio de no-arbitrariedad el que puede universalizarse, solo dicho principio es el resquicio mínimo de ese programa ambicioso del racionalismo clásico. La tensión en FMQ se presenta justamente por esa disyuntiva: el racionalismo no puede sostenerse ante un contexto tan adverso como el que hemos comentado anteriormente, pero tampoco está en disposición para aceptar su final definitivo. Entonces aparece la respuesta tímida, pero honesta: no se salva el racionalismo clásico, pero sí un tipo especial de racionalismo. Un racionalismo que conserva "un mínimo de universalidad y necesidad" como instrumento fundamental del conocimiento científico. Si la premisa es que, de vencer el escepticismo, es la filosofía misma la que conocería su final, entonces no puede negarse alguna forma de sobrevivencia del racionalismo. 
Así lo concibe FMQ y establece que ese tipo de racionalismo que sobrevive es "la creencia de que, aunque de manera imperfecta, la razón permite fundamentar un campo muy grande, y siempre en aumento, de conocimientos cuyo poder suasorio es prácticamente universal” (Miró Quesada, 2012f, p. 126).

Otro aspecto que es importante considerar para entender por qué nos enfrentamos a una cuestión más pragmática y vital que meramente teórica, es ver el lugar que ocupan las disquisiciones sobre la razón dentro del sistema de pensamiento de FMQ. En un texto de 1986 titulado "La filosofía como actividad racional", FMQ confiesa muy sintéticamente algo que lo ha expresado en diferentes otros textos. Dice que todo el problema de la razón y del conocimiento es "solo un procedimiento metodológico" (Miró Quesada, 2012e, p. 97). Es decir, que el problema de la razón nos prepara para el verdadero problema de la filosofía, que no es otro que el problema del hombre. La filosofía toda se resume cuando decimos que ella es la posibilidad misma de preguntarnos por el sentido de la vida, del mundo, del ser humano y de Dios. Las preguntas epistemológicas, es decir, aquellas que nos enfrentan al problema de la posibilidad de alcanzar respuestas universales y objetivas por parte de la razón, no son sino preguntas metodológicas que nos ayudan a reconocer nuestros reales límites ante las otras preguntas antropológicas más fundamentales. El problema en el fondo no era pues el problema de la razón, este era solo un paso previo para encarar luego la pregunta vital. Una respuesta negativa ante el problema de la razón, cierra toda posibilidad de desarrollo para la cuestión antropológica y entonces la filosofía pierde sentido y la vida misma del filósofo se perjudica con ella.

No obstante, al escéptico le queda todavía un recurso. Si bien la filosofía puede conocer su final con el derrumbe de la razón, el escéptico podría alegar que tal situación no necesariamente determina un mundo peor. En el texto en el que Richard Rorty comenta sobre la posibilidad de un mundo posfilosófico, también se hacía esa misma pregunta. ¿Por qué deberíamos de pensar que un mundo sin filosofía es necesariamente peor? Cabe la posibilidad, decía Rorty, que ese mundo posfilosófico fuese enormemente superior al mundo que enfrentamos hoy. Pues 
bien, en el mundo de lo posible cualquier alternativa es válida; pero para FMQ, más allá de que sea una alternativa válida, el fin de la filosofía representa una real pérdida para la sociedad. La filosofía es, según nuestro autor, "la garantía del sentido de la historia" (Miró Quesada, 2012e, p. 92).

Para FMQ, la filosofía era "la lucha indoblegable por humanizar el mundo" (Miró Quesada, 2012e, p. 103), por lo tanto no es un simple paso el dejarla. La filosofía cumple un rol humanizador y liberador. Frente a la cuadratura de una vida impuesta por parámetros y la burocracia, la filosofía se convierte en la esperanza de una vida conducida por la razón; una vida guiada por principios inobjetables que salvaguardan la humanidad. Una vida sin filosofía es la condena a vivir a expensas de la fuerza, la manipulación o los automatismos. Entonces, claro que no podía FMQ ceder en la lucha con los escépticos, pues en esa lucha se jugaba la esperanza en el futuro de la sociedad.

Resumiendo, lo que he tratado de hacer es revelar una tensión vital en FMQ. Por un lado, un contexto que lo obliga a aceptar el fin del racionalismo clásico, el fin de las pretensiones nobles de la razón; es decir, reconocer la imposibilidad de un conocimiento absoluto. Mas por otro lado, la necesidad de no dejarse vencer ante el escepticismo, pues eso significaría el fin de la filosofía. La respuesta de nuestro filósofo es un nuevo tipo de racionalismo mucho más tímido que él reconoce como 'mínimo', en el que aunque sea un principio se salva. Ese racionalismo mínimo por lo menos es la garantía de que aún podemos tener la esperanza en la razón y en la posibilidad de una teoría de los principios.

\section{La defensa de una razón limitada y pragmática}

En lo que sigue, sin embargo, me pondré frente a FMQ y defenderé aquello que creo nuestro filósofo no se atrevió a concluir: la sobrevivencia de una razón limitada y pragmática. En la tensión que he mostrado, FMQ tiene claro que hay muchos motivos para reconocer un posible derrumbe de la razón, pero también le es claro que todo ese contexto escéptico no puede vencer por completo y que debe salvarse por lo menos una razón mínima. Yo creo, en cambio, que lo que debe salvarse no es esa razón mínima que debemos encontrar, sino más bien el tipo de razón que se 
nos ha revelado tras la investigación; es decir, la razón limitada y pragmática. En su intento por defender la razón, en esa lucha de 50 años contra el escepticismo, lo que aparece evidente son más bien los límites de la razón y la profundidad de los argumentos pragmáticos. Muchas de esas razones que parecieron ser trascendentales fueron en verdad pragmáticas, y eso nos lleva a concluir que quizá las razones pragmáticas son el fondo y carácter propio de la razón.

Creo que haber elegido el camino de búsqueda de una razón mínima no fue realmente una respuesta a la profunda revolución que se estaba produciendo en la cultura occidental. Cuando Miró Quesada se encuentra con el texto de Kurt Gödel, reconoce la real revolución que este puede causar a nuestra comprensión de la razón. Gödel revela el drama del formalismo que no puede nunca abarcar todo lo que los procesos intuitivos son capaces de lograr. Realmente era una revolución profunda al interior de las esperanzas racionalistas configuradas desde Platón; toda una tragedia para el formalismo, pero la conclusión que obtiene no lo guía hacia la razón intuitiva, que era el tipo de razón que se había revelado como la más sólida, sino que más bien ve la defensa de un principio mínimo, el de no-arbitrariedad:

El formalismo no puede mantenerse porque, tal como hemos mostrado, uno de los resultados fundamentales del teorema de Kurt Gödel es que la matemática no puede formalizarse totalmente. Y si no puede formalizarse totalmente, no puede reducirse a un puro lenguaje. Pero si no puede reducirse a un puro lenguaje no puede mostrarse que las reglas de inferencia son arbitrarias. (Miró Quesada, 2012d, p. 139)

Releyendo la cita, se descubre más bien el drama de FMQ, la tensión que he buscado mostrar: su necesidad de salvar ese mínimo de la razón que, sin embargo, todavía es concebida bajo los parámetros clásicos. La razón mínima es, en el fondo, un cambio cuantitativo, que busca salvar por lo menos algún principio correspondiente a la razón. La configuración de una razón mínima, que es el camino elegido por FMQ, no lo lleva a superar el modelo clásico, sino que más bien salva dicho modelo con una medida mínima. FMQ no reconoce que con el drama del formalismo se revele también el drama del modelo fundacionalista 
de la razón, el modelo que pretendió una razón autónoma y completamente transparente en sus principios. Lo que se nos ha revelado es la imposibilidad de fundamentar autónomamente la razón y, al mismo tiempo, la imposibilidad de una trasparencia plena de la razón.

Como argumenté en la segunda sección, la motivación principal de FMQ era en el fondo más pragmática que teórica. Si su motivación no hubiese sido tan vital, quizá la pregunta que hubiese enfrentado no fuese “¿cuál es el principio que se salva del modelo clásico?”, sino más bien ¿por qué debemos de pensar que existen principios trascendentales de la razón? y ¿por qué debemos de pensar que tales principios pueden ser conocidos? En el fondo nos hubiésemos preguntado primero si son los principios los que anteceden a la razón humana o son aquellos productos de la propia razón, es decir, ¿la razón se nutrió de esos principios o la razón fue apareciendo con estos principios?

Mi propuesta, más bien, se encamina en una ruta contraria de la asumida por FMQ. Yo cada vez estoy más convencido de que el carácter principal de la razón se describe mejor cuando afirmamos que esta es limitada y pragmática, y que, sin embargo, dicha explicación no significa el fin de la razón. Cuando afirmo el carácter limitado de la razón, no digo solo que esta es situada, y por tanto condicionada, y que dichas condiciones revelan límites. Más allá de eso, quiero decir que la manera en que la razón se manifiesta es justamente a través de esos límites. ¿Acaso no hemos escuchado siempre que la necesidad afina nuestra inteligencia? Pues bien, esa es mucho más que solo una expresión de la sabiduría popular, es en realidad un enunciado que revela el carácter mismo de la razón. Creo que es un camino errado pretender comprender la razón humana entendiéndola como dada (Kalpokas, 2010) o como trascendental a las vicisitudes de la persona en su contexto determinado. Pero ese camino errado es el que se ha pretendido constantemente y es el trasfondo de la empresa racionalista que el propio FMQ también trató de defender. La razón dada es en realidad un resquicio de los modelos creacionistas que niegan la visión evolutiva de toda la naturaleza. Supone pensar que ella, la razón, es un ente metafísico trascendental que guarda 
en sí todas las verdades o el sentido de todo lo correcto y que sirve de último criterio de lo que debe ser nuestra vida concreta. El proyecto racionalista se enfrascó en la búsqueda de los principios básicos de dicha razón para hacerla más a nuestro alcance, pero al sentir toda la objeción de los modelos kantianos, en cuanto a las posibilidades de la propia razón para autorreflejarse, una salida poskantiana fue rebuscar en el corazón mismo de las matemáticas esa respuesta. En gran medida, el proyecto de FMQ bebe de la misma expectativa que animó los trabajos de Russell, Frege o el primer Husserl: transparentar los principios básicos de la razón a partir del análisis de los fundamentos de las matemáticas. Mi forma de encarar el problema es distinta. Me siento más en deuda con autores como Charles Peirce, John Dewey, William James, Charles Darwin, el segundo Wittgenstein o Thomas Kuhn, y creo que lo que ellos nos han enseñado es que a la razón se la entiende en su ejercicio y no fotográficamente. En otras palabras, si nuestro interés es reconocer los principios de la razón, estos no pueden ser buscados de manera a priori, pues ellos se han ido presentando de manera evolutiva como respuesta a las urgencias concretas de la vida del ser humano en una naturaleza que le fue hostil y desafiante.

Filogenéticamente hablando, eso que acabo de manifestar quiere decir que la razón humana es, en sus principios fundamentales y en toda su expresión, un resultado de la evolución humana (Quintanilla, Mantilla y Cépeda, 2014); y como todo producto de la evolución el trasfondo es siempre pragmático, es decir, con el único fin de hacer más asequible la vida misma. En mi opinión, no hay diferencia sustancial entre la formación de las uñas de nuestros dedos y la formación evolutiva de nuestra razón. En ambos casos nos encontramos ante salidas "inteligentes" de la evolución desde necesidades concretas. Entiendo, sin embargo, que para muchos puede sonar ofensivo eso que acabo de decir, pues supone una desmitificación de nuestra razón y de su superioridad por sobre todo el resto de nuestro cuerpo, sus órganos y sus funciones. Pero es necesario reconocer que esa mitificación de la razón, esa supuesta trascendentalidad de la misma, es solo producto de una idiosincrasia moderna que la filosofía forjó a partir de una disociación entre el sujeto y el objeto. Claro que no fue solo y exclusivamente 'moderna' tal idiosincrasia, pues podríamos encontrar indicios de 
ella en la filosofía griega, por ejemplo, en la filosofía de Platón. Pero es evidente que es en la obra de Descartes donde se escinde con claridad al yo que piensa y al cuerpo que trabaja en el mundo. Es a partir de tal escisión por lo que la razón se nos revela con absoluta trascendentalidad y se consolida la idea de su versión como dada, acabada y ajena a la vida misma. En el fondo, la lucha de Darwin y el evolucionismo contra la idea de especies fijas e inmutables traía consigo una serie de repercusiones epistemológicas que la filosofía poco a poco está reconsiderando (Dewey, 1994). Entre esas repercusiones, por supuesto, hay que sopesar la nueva forma de entender la razón y su supuesta trascendentalidad. La mirada evolucionista nos obliga a reconocer que el sendero del desarrollo es uno y el mismo, como dije antes, tanto para las uñas como para la razón; y es por ello que puedo atreverme a decir que no hay diferencias sustanciales entre la formación de las uñas y el desarrollo de nuestra razón.

Se puede objetar mi afirmación aduciendo que estoy suponiendo un progreso 'inteligente' o en todo caso teleológico. Entonces, la pregunta sería ¿con qué criterios se podría reconocer ese desarrollo que va de menos a más? De hecho, hay muchas discusiones sobre el tema, discusiones a las que yo no deseo entrar en este texto. Podría darse el caso de que no todo el proceso evolutivo fuera para mejor, pero creo que eso no invalida mi afirmación sobre el desarrollo de la razón y su indiferencia sustancial con el crecimiento de las uñas en nuestros dedos. Podría quizá objetar mi afirmación de que el carácter sustancial de la razón es su fin pragmático, pero no necesariamente; pues si consideramos que ese carácter pragmático no siempre debe reconocerse como encaminado hacia lo mejor (pues en la praxis no conocemos necesariamente cuál es el mejor camino y solo contamos con nuestros recursos para elegirlo), entonces tampoco queda comprometida mi afirmación de un desarrollo pragmático de la razón con un supuesto telos inteligente. En otras palabras, que la razón sea pragmática no garantiza que nuestra elección siempre sea la mejor; de hecho, los criterios pragmáticos solo nos empujan a reconocer la complejidad de nuestra elección, pero no a pretender que sus decisiones son inteligentes porque son mejores. 
Deseo afianzar aún más mi argumento y para ello añadiré ahora una reflexión ontogenética de clara raigambre piagetiana. Consideremos, pues, el desarrollo de la razón en el ser humano, desde la niñez y su camino hasta la adultez. Nuevamente deseo mostrar que en dicho desarrollo lo que se revela es un carácter pragmático y limitado de esta. El esquema básico sobre el cual parece haberse manifestado la razón a lo largo del recorrido se nos presenta como partiendo de una necesidad, movido por un interés y alcanzando un punto de satisfacción flexible en el cual pragmáticamente se puede reconocer una superación de la urgencia práctica. Tal esquema es posible reconocerlo en el infante cuando construye su espacialidad en su primer año de vida, por la formación de los significados en los niños entre los 2 y 3 años, los escolares que elaboran su aprendizaje o los adolescentes que resuelven problemas abstractos. En todos estos casos, el esquema es casi el mismo. El punto de partida siempre es una situación que se percibe como una urgencia de carácter práctico (la necesidad de desplazarse, la necesidad de la comunicación o el conflicto cognitivo creado por el profesor). Por lo mismo, parece claro también que siempre hay una motivación interesada.

Como afirman los expertos, ninguno de esos movimientos es gratuito. El motor de todos ellos es la necesidad de transformar una situación limitante o hacer más llevadera una exigencia circunstancial. Entonces el individuo se ve interesado por el tipo de resultado que obtendrá (Priniski et ál., 2019). Por supuesto, el interés va unido a expectativas y a una consideración flexible de la satisfacción. ¿Cómo podríamos negar estos elementos como conformantes de la razón? ¿Por qué pensar que solo son principios de la razón aquellos que aparecen como principios de la lógica o de la matemática? John Dewey afirma que tal tendencia en la filosofía deriva de un menosprecio de esta hacia el interés o hacia lo interesado debido a un hábito que se formó desde el mundo griego. El interés está asociado a lo manual, a lo práctico, a lo no-sublime o hacia lo mundano, que para el mundo griego era lo menos digno dentro de lo social (Dewey, 1995). Si la vida del individuo nos demuestra lo contrario a ese hábito de la filosofía y más bien vemos un desarrollo de la razón siempre a partir del interés, entonces solo parece obnubilación negar que el interés es parte de la estructura esencial de la razón. 
Finalmente, he hecho referencia a la satisfacción flexible como punto de llegada (causa final) para asumirlo como parte final de ese esquema de la razón que estoy proponiendo. Esta idea de un punto de satisfacción flexible es otro término para indicar que el carácter mismo de la razón es adaptativo. Esto último no significa solo que la razón se acomoda a sus condicionantes, sino que además, adaptándose a sus limitaciones o incluso obteniendo provecho de ellas, las expectativas se adaptan y así el punto de llegada se va haciendo. Es pues una postura que niega toda posibilidad de una causa final que estuviera configurada antes de iniciar el proceso mismo del ejercicio de la razón, es decir, se trata de un desarrollo sin telos. Parafraseando la célebre metáfora de Otto Neurath: el barco lo vamos recomponiendo en la misma marcha.

\section{Conclusión}

Lo que he tratado de mostrar, especialmente en la anterior sección, no es una propuesta opuesta a la de FMQ, sino más bien una propuesta que se arriesgue a dar ese último paso que nuestro filósofo no quiso dar del todo. Creo que FMQ recorrió un sendero que lo acercaba hacia este mismo fin, pero - como mostré en la primera parte - luchó tensamente contra ese final. Para mí es elocuente la siguiente cita en la que aparece un FMQ reconociendo esa dinamicidad de la razón, pero al mismo tiempo la incapacidad de la filosofía para asirla, para tratarla en toda su complejidad e inestabilidad: "Todo nos obliga a aceptar que existe una dinámica de la razón, una manera de funcionar del pensamiento racional que, debido a su profundidad y a la falta de medios analíticos adecuados, ha escapado a la comprensión filosófica" (Miró Quesada, 2012d, p. 397).

Me quedó al final una cuestión por discutir y es que, como dijimos, la negativa de FMQ para dar ese paso hacia el reconocimiento de una razón limitada va acompañada del temor a que ello signifique el fin de la filosofía y la victoria del escepticismo. En realidad, la respuesta a dicha cuestión planteada supondría un libro completo en el que se pudiera hilar la situación de una nueva filosofía, acompañada de un sano escepticismo, pero avivada por nuevos hábitos y nuevas expectativas. Por tanto, debo decir que no creo que ese paso que yo doy hacia una 
razón pragmática y limitada pueda necesariamente significar el fin de la filosofía, tal vez sí el fin de la filosofía tal y como la conocemos, pero también quizá la apertura hacia una nueva filosofía basada esta vez en una epistemología evolutiva y pragmatista. Lo que sí puedo asegurar es que esta también es una apuesta, pues cuento con argumentos que me impulsan a reconocer esa nueva forma de epistemología (y su contraparte de filosofía), pero no creo tampoco que dichos argumentos sean definitivos o que nos garanticen un camino evidente y seguro; todo lo contrario, creo que esta definición es una apuesta con algún grado de incertidumbre, pero ¿por qué no aprender a convivir con un poco de incertidumbre?

\section{Referencias bibliográficas}

Dewey, J. (1994). The Influence of Darwinism on Philosophy. En J. Gouinlock (Ed.), The Moral Writings of John Dewey (pp. 101-119). Nueva York: Prometheus Books.

Dewey, J. (1995). El interés y la disciplina. En Democracia y educación. Una introducción a la filosofía de la educación (pp. 112-123). Madrid: Morata.

Husserl, E. (2008). La crisis de las ciencias europeas y la fenomenología trascendental. Buenos Aires: Prometeo Libros.

Kalpokas, D. (2010). Dewey y el mito de lo dado. Endoxas: Series filosóficas, 26, 157-186. doi: 10.5944/endoxa.26.2010.30

Marías, J. (1964). La crisis de la física clásica. La ciencia actual. En Historia de la filosofia y de la ciencia (pp. 329-363). Madrid: Ediciones Guadarrama.

Miró Quesada, F. (1966). Filosofía rigurosa y filosofía literaria. Alpha, 2(5), 1-5.

Miró Quesada, F. (2012a). Esbozo de mi posición filosófica (1953). En Obras Esenciales. Tomo I. Autoexposiciones y autobiografias intelectuales. Sentido del Movimiento Fenomenológico (pp. 89-94). Lima: Universidad Ricardo Palma.

Miró Quesada, F. (2012b). Crisis de la ciencia y teoría de la razón (1958). En Obras Esenciales. Tomo III, Vol. 1. Apuntes para una teoría de la razón. Textos conexos (pp. 371-384). Lima: Universidad Ricardo Palma. 
Miró Quesada, F. (2012c). Apuntes para una teoría de la razón (1962). En Obras Esenciales. Tomo III, Vol. 1. Apuntes para una teoría de la razón. Textos conexos (pp. 385-405). Lima: Universidad Ricardo Palma.

Miró Quesada, F. (2012d). Apuntes para una teoría de la razón (1963). En Obras Esenciales. Tomo III, Vol. 1. Apuntes para una teoría de la razón. Textos conexos (pp. 23-356). Lima: Universidad Ricardo Palma.

Miró Quesada, F. (2012e). La filosofía como actividad racional (1986). En Obras Esenciales. Tomo I. Autoexposiciones y autobiografias intelectuales. Sentido del Movimiento Fenomenológico (pp. 95-104). Lima: Universidad Ricardo Palma.

Miró Quesada, F. (2012f). Autobiografía filosófica. 1994 o 1995. En Obras Esenciales. Tomo I. Autoexposiciones y autobiografías intelectuales. Sentido del Movimiento Fenomenológico (pp. 115-128). Lima: Universidad Ricardo Palma.

Miró Quesada, F. (2012g). Esquema para una teoría de la razón. Ensayo de fundamentación racionalista (2012). En Obras Esenciales. Tomo III, Vol. 2 (pp. 23-201). Esquema para una teoría de la razón. Textos conexos. Lima: Universidad Ricardo Palma.

Ortega y Gasset, J. (1966). Relativismo y racionalismo. En Obras Completas, tomo III (1917-1928), 6a . edición (pp. 157-162). Madrid: Revista de Occidente.

Priniski, S., Rosenzweig, E., Canning, E., Hecht, C., Tibbetts, Y. \& Harackievicz, M. (2019). The Benefits of Combining Value for the Self and Others in Utility-Value Interventions. Journal of Educational Psichology. 111(8). doi: $10.1037 / \mathrm{edu} 0000343$

Quintanilla, P., Mantilla, C. y Cépeda, P. (2014). Evolución y desarrollo de la cognición social. En: Cognición social y lenguaje. La intersubjetividad en la evolución de la especie y en el desarrollo del niño. Lima: Pontificia Universidad Católica del Perú.

Reichenbach, H. (1967). La filosofía científica. Ciudad de México: Fondo de Cultura Económica.

Rorty, R. (1996). Consecuencias del pragmatismo. Madrid: Tecnos.

Wittgenstein, L. (1988). Investigaciones filosóficas. Barcelona: Editorial Crítica. 